\title{
Impact of Age, Rurality and Distance in Predicting Contralateral Prophylactic Mastectomy for Breast Cancer in a Midwestern state: A Population-Based Study
}

Ingrid Lizarraga ( $\Delta$ ingrid-lizarraga@uiowa.edu )

University of lowa Hospitals and Clinics https://orcid.org/0000-0001-9376-1746

Amanda R Kahl

University of lowa

Ellie Jacoby

Drake University

Mary E Charlton

University of lowa

Charles F Lynch

University of lowa

Sonia L Sugg

University of lowa

\section{Research article}

Keywords: contralateral prophylactic mastectomy (CPM), median travel distances (MTD)

Posted Date: August 14th, 2020

DOI: https://doi.org/10.21203/rs.3.rs-54758/v1

License: (c) (i) This work is licensed under a Creative Commons Attribution 4.0 International License. Read Full License 


\section{Abstract}

Background: There is substantial variability in the use of contralateral prophylactic mastectomy (CPM) in women with unilateral breast cancer across the United States. lowa is one of several rural Midwestern states found to have the highest proportions of CPM nationally in women $<45$ years of age. We evaluated the role of rurality and travel distance as factors related to these surgical patterns.

Methods: Women with unilateral breast cancer (2007-2017) were identified using lowa Cancer Registry records. Patients and treating hospitals were classified as metro, nonmetro and rural based on Rural-Urban Continuum Codes. Differences in patient, tumor, and treatment characteristics and median travel distances (MTD) were compared. Characteristics associated with CPM were evaluated with multivariate logistic regression.

Results: 22,158 women were identified: $57 \%$ metro, $26 \%$ nonmetro and $18 \%$ rural. The overall proportion of CPM in lowa was consistently higher than in the national Surveillance, Epidemiology, and End Results (SEER18) throughout the interval from 2007-2015. Young rural women had the highest proportion of CPM (<40 years: $52 \%, 39 \%$ and $40 \%$ for rural, metro, nonmetro, respectively). Half of all rural women had surgery at metro hospitals; these women had the longest MTD (56 miles). Of all women treated at metro hospitals, rural women had the highest proportion with CPM (17\% rural; vs $14 \%$ metro/nonmetro, $p=0.007$ ). On multivariate analysis, traveling $\geq 50$ miles (ORs $1.48-2.34$ ) or being rural regardless of travel distance was predictive of $\mathrm{CPM}(\mathrm{OR}=1.36)$. Other risk factors were young age $(<40$ years: $\mathrm{OR}=7.18$, $95 \% \mathrm{Cl}: 5.89-8.76)$ and surgery at a metro hospital that offers reconstruction (OR=2.3, 95\% Cl: $1.70-3.21)$ and is not $\mathrm{NCl}$ designated (OR=2.19, 95\% Cl: 1.78-2.69).

Conclusion: There is an unexpectedly high proportion of CPM use in young rural women in lowa. Travel $\geq 50$ miles and rural residence are independently associated with likelihood of CPM. Disparities in access to specialty care may underlie the desire for surgery that is perceived to minimize follow-up visits.

\section{Background:}

Surgical treatment for unilateral breast cancer includes mastectomy and lumpectomy with radiation, both of which are considered equally efficacious [1]. Contralateral prophylactic mastectomy (CPM), or removal of the unaffected breast, has become increasingly common nationally [2] despite the lack of survival benefit in average risk women [3]. According to multiple national guidelines, CPM should be discouraged in average risk women [4-6].

The US Midwest was recently noted to have a high proportion of CPM use with the state of lowa having the second highest proportion nationally (44.9\%) among women aged 20-44 during 2010-2012 [7, 8]. The etiology for this trend in the Midwest was unclear. Like lowa, most of the Midwest is agricultural with over half of Midwestern counties considered rural [9]. Rurality has been shown to be associated with disparities in breast cancer throughout its spectrum of care [9-13]. Rural women are more likely to receive mastectomy and less likely to receive reconstruction, radiation or chemotherapy [14-17]. The impact of rurality on breast cancer disparities and specifically surgical treatment could reflect a number of factors, including travel distance, socioeconomic and educational status, and access to multidisciplinary cancer care $[18,19]$. Women who travel further for surgical care were shown to be more likely to undergo CPM in the National Cancer Database (NCDB), although the relationship between travel distance and rurality was not studied [7].

lowa is a rural state with a high-quality statewide cancer registry. Considering the reported high proportion of CPM, we analyzed patterns of breast cancer care in detail to examine the possible causes. We hypothesized that use of CPM may be partially associated with patient travel distance for treatment and access to breast cancer health care resources. 


\section{Methods:}

Women with unilateral breast cancer (2007-2017) were identified using lowa Cancer Registry records. Patients and treating hospitals were classified as metro, nonmetro and rural based on Rural-Urban Continuum Codes. Differences in patient, tumor, and treatment characteristics and median travel distances (MTD) were compared. Characteristics associated with CPM were evaluated with multivariate logistic regression.

\section{Results:}

22,158 women were identified: $57 \%$ metro, $26 \%$ nonmetro and $18 \%$ rural. The overall proportion of CPM in lowa was consistently higher than in the national Surveillance, Epidemiology, and End Results (SEER18) throughout the interval from 2007-2015. Young rural women had the highest proportion of CPM (< 40 years: $52 \%, 39 \%$ and $40 \%$ for rural, metro, nonmetro, respectively). Half of all rural women had surgery at metro hospitals; these women had the longest MTD (56 miles). Of all women treated at metro hospitals, rural women had the highest proportion with CPM (17\% rural; vs $14 \%$ metro/nonmetro, $p=0.007$ ). On multivariate analysis, traveling $\geq 50$ miles (ORs 1.48-2.34) or being rural regardless of travel distance was predictive of CPM $(\mathrm{OR}=1.36)$. Other risk factors were young age $(<40$ years: $\mathrm{OR}=7.18,95 \% \mathrm{Cl}$ : $5.89-8.76)$ and surgery at a metro hospital that offers reconstruction ( $\mathrm{OR}=2.3,95 \% \mathrm{Cl}: 1.70-3.21)$ and is not $\mathrm{NCl}-$ designated $(\mathrm{OR}=2.19,95 \% \mathrm{Cl}: 1.78-2.69)$.

\section{Conclusion:}

There is an unexpectedly high proportion of CPM use in young rural women in lowa. Travel $\geq 50$ miles and rural residence are independently associated with likelihood of CPM. Disparities in access to specialty care may underlie the desire for surgery that is perceived to minimize follow-up visits.

\section{Methods}

\section{Data Source and Study Population}

A secondary data analysis of lowa breast cancer patients was performed. Cases were extracted from the lowa Cancer Registry Surveillance, Epidemiology, and End Results (SEER) Data Management System (SEER*DMS) using the following inclusion criteria: lowa women with unilateral, first primary, stage 0-III breast cancer (AJCC 6th edition) diagnosed between 2007 and $2017(n=23,210)$. Patients were excluded if they had synchronous breast cancers $(n=$ 699 ) or lobular carcinoma in situ (ICD-0-3 histology code 8520/2; $n=353$ ). This study was granted human subject exemption status by the University of lowa Institutional Review Board.

\section{Study Variables}

Patient demographics in the dataset included age at diagnosis, race, marital status, insurance, year of diagnosis, and patient residence at diagnosis. Patients' county of residence and treating hospital locations were categorized based on the US Department of Agriculture's Rural-Urban Continuum Codes (RUCC), which categorize counties based on metropolitan areas within the county and the degree of urbanization in adjacent counties (Fig. 1) [20]. lowa metropolitan (metro) counties included RUCC 1-3, nonmetro 4-6, and rural 7-9, as used elsewhere [21]. Nonmetro counties contain cities with $\geq 20,000$ people and/or are adjacent to counties containing metro areas, whereas rural counties are not adjacent to counties containing metro areas and/or only have cities with $<2,500$ people. A geographic analysis of our rural category revealed that patients in these counties, on average, would have to travel $\geq 50$ miles to the closest Commission on Cancer (CoC) hospital (data not shown). 
County-level patient demographics of poverty level and percentage of county with bachelor's degree or higher from the 2016 American Community Survey were included [22]. Tumor characteristics included stage, lymph node status, histology, and breast cancer subtype. Treatment characteristics included primary surgery site (unilateral mastectomy (UM) included surgery codes $30,40-41,43-46,50-51,53-56,61,64-67,70-71,80$; CPM 42, 47-49, 52, 57-59, 6263, 68-69, 72-75; lumpectomy 20-24; none/other/unknown included all other surgery codes), and breast

reconstruction surgery (created from the surgery variable; reconstruction performed included codes $43-49,53-59,63-$ $69,73-75)$ [23].

Characteristics of the surgery hospital were linked to patients and included rurality; CoC accreditation status and $\mathrm{NCl}$ cancer center designation; bed size; availability of reconstruction services (derived by identifying any facility in the cohort that had provided reconstruction surgery between 2007-2017); and travel distance to surgery facility, which was calculated with Great Circle Distance in ArcGIS utilizing latitudes and longitudes of hospital location and patient residence at the time of cancer diagnosis. There were 1,585 patients ( 877 metro, 437 nonmetro, and 271 rural patients) who did not match to a surgery hospital and hence are missing from the surgery hospital variables. This most commonly occurred when surgery was performed at a non-hospital facility such as an ambulatory surgery center.

Figure 1. Map of lowa Hospitals and County Rurality

\section{Statistical Analysis}

Rates were calculated using SEER ${ }^{*}$ Stat, version 8.3.5 [24]. Differences in patient, tumor, and treatment characteristics were compared between patient and hospital rurality groups using Chi-square tests. A multivariable logistic regression was used to determine the characteristics associated with CPM compared to other surgery types. All variables were included in a stepwise selection model with an exit significance level of 0.2 and the significant variables were included in the final model. All variables listed above were considered for inclusion into the model. ANOVA analysis was performed to compare median travel distance between patient rurality groups. Analyses were conducted in SEER*Stat, SAS 9.4 (SAS Institute, Cary, NC) and ArcGIS 10.5.1 (ESRI, Redlands, CA). P-values $<0.05$ were considered statistically significant. Patients with missing relevant variables were excluded from the models.

\section{Results}

The study cohort consisted of 22,158 women (Table 1). Over half (57\%) of the women lived in metro, $26 \%$ in nonmetro and $18 \%$ in rural counties. Regardless of residence, two-thirds were married, $90 \%$ had private or Medicare insurance, and nearly two-thirds had early stage disease (stage 0 or 1 ). Overall, $60 \%$ had lumpectomy, $25 \%$ had UM, and $13 \%$ had CPM. 
Table 1

Patient, tumor, and treatment characteristics by patient's residence at time of diagnosis, lowa, 2007-2017

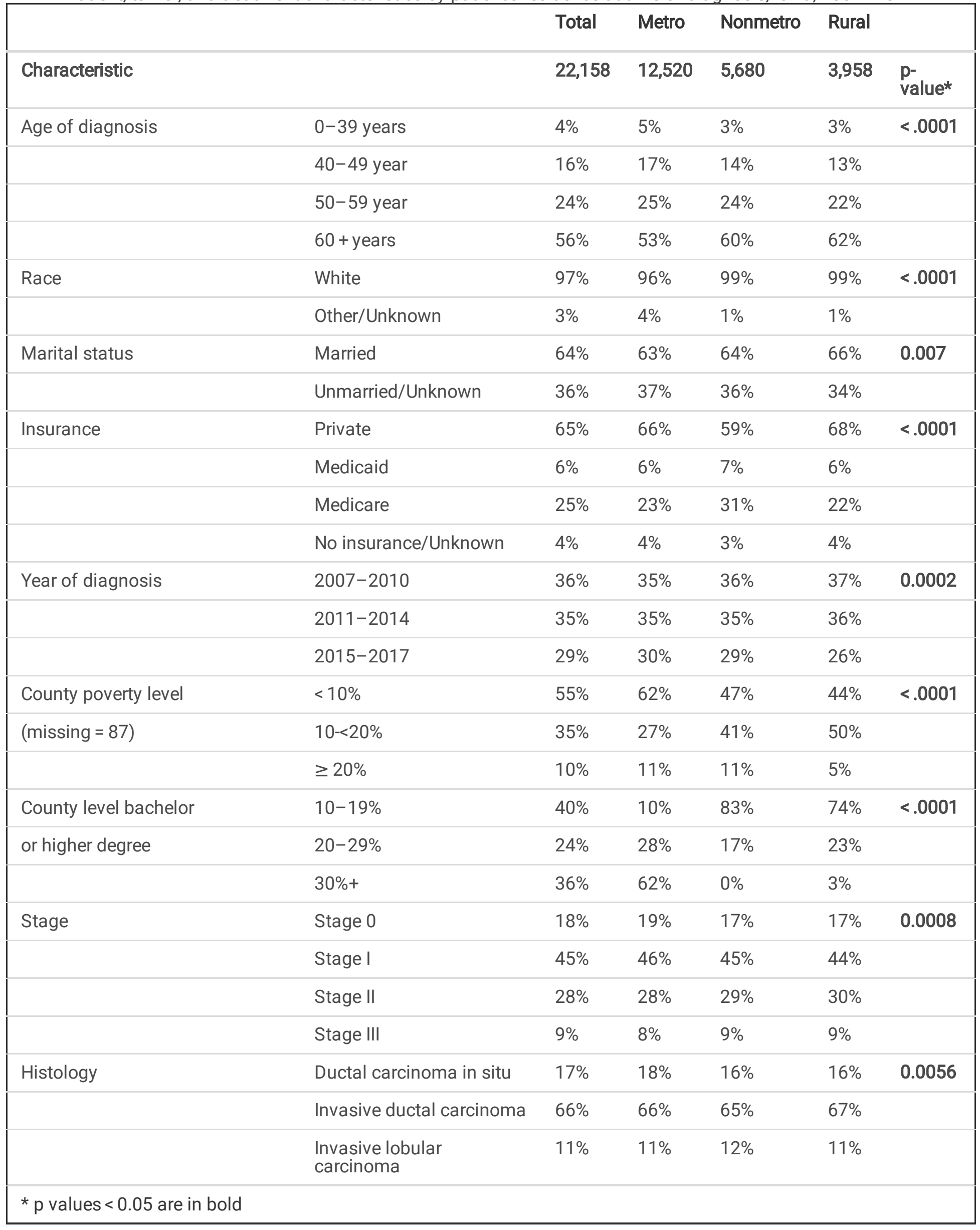




\begin{tabular}{|c|c|c|c|c|c|c|}
\hline & & Total & Metro & Nonmetro & Rural & \\
\hline & Other & $6 \%$ & $6 \%$ & $6 \%$ & $6 \%$ & \\
\hline \multicolumn{7}{|l|}{ Surgery Categorizations } \\
\hline \multirow[t]{4}{*}{ Surgery type } & CPM & $13 \%$ & $14 \%$ & $11 \%$ & $13 \%$ & $<.0001$ \\
\hline & Unilateral mastectomy & $25 \%$ & $24 \%$ & $27 \%$ & $27 \%$ & \\
\hline & Lumpectomy & $60 \%$ & $60 \%$ & $60 \%$ & $58 \%$ & \\
\hline & None/Other/Unknown & $2 \%$ & $2 \%$ & $3 \%$ & $2 \%$ & \\
\hline \multirow[t]{5}{*}{ Reconstruction by surgery type } & $\mathrm{CPM}+$ reconstruction & $7 \%$ & $7 \%$ & $5 \%$ & $6 \%$ & $<.0001$ \\
\hline & CPM only & $6 \%$ & $6 \%$ & $6 \%$ & $7 \%$ & \\
\hline & $\mathrm{UM}+$ reconstruction & $3 \%$ & $3 \%$ & $2 \%$ & $2 \%$ & \\
\hline & UM only & $22 \%$ & $20 \%$ & $25 \%$ & $24 \%$ & \\
\hline & Lumpectomy & $62 \%$ & $63 \%$ & $62 \%$ & $60 \%$ & \\
\hline Reconstruction after any & Yes & $9 \%$ & $11 \%$ & $7 \%$ & $9 \%$ & $<.0001$ \\
\hline mastectomy & No & $91 \%$ & $89 \%$ & $93 \%$ & $91 \%$ & \\
\hline $\begin{array}{l}\text { Age at Diagnosis by Surgery } \\
\text { Type }\end{array}$ & $0-39$ years $+C P M$ & $41 \%$ & $39 \%$ & $40 \%$ & $52 \%$ & 0.022 \\
\hline \multirow[t]{7}{*}{ (no surgery excluded) } & $0-39$ years + non $-\mathrm{CPM}$ & $59 \%$ & $61 \%$ & $60 \%$ & $48 \%$ & \\
\hline & $40-49$ year + CPM & $25 \%$ & $26 \%$ & $21 \%$ & $30 \%$ & 0.0016 \\
\hline & 40-49 year + non-CPM & $75 \%$ & $74 \%$ & $79 \%$ & $70 \%$ & \\
\hline & $50-59$ year + CPM & $15 \%$ & $15 \%$ & $14 \%$ & $17 \%$ & 0.1653 \\
\hline & $50-59$ year + non-CPM & $85 \%$ & $85 \%$ & $86 \%$ & $83 \%$ & \\
\hline & $60+$ years $+C P M$ & $7 \%$ & $7 \%$ & $6 \%$ & $7 \%$ & 0.0271 \\
\hline & $60+$ years + non-CPM & $93 \%$ & $93 \%$ & $94 \%$ & $93 \%$ & \\
\hline \multirow[t]{3}{*}{ Surgery Hospital Location } & Metro & $80 \%$ & $99 \%$ & $59 \%$ & $50 \%$ & $<.0001$ \\
\hline & Nonmetro & $14 \%$ & $0 \%$ & $40 \%$ & $21 \%$ & \\
\hline & Rural & $6 \%$ & $0 \%$ & $2 \%$ & $29 \%$ & \\
\hline
\end{tabular}

The overall rate of CPM in lowa was consistently higher than in SEER18 throughout the interval from 2007-2014 (Fig. 2). When examined by patient residence, the rate for nonmetro women follows the national trend. For metro and rural women however, the rates were persistently higher between 2007-2014, peaking in 2013, with a trend toward convergence with national rates after 2014. The proportion of CPM was significantly higher for both rural (13\%) and metro $(14 \%)$ women compared to nonmetro women $(11 \%)$. Young rural women had the highest proportion of CPM use, with $52 \%$ of rural women age $<40$ years undergoing CPM compared to $40 \%$ and $39 \%$ of metro and nonmetro women, respectively. Of those who received $\mathrm{UM}, 12 \%$ had reconstruction, while of those who had $\mathrm{CPM}, 50 \%$ underwent reconstruction. 


\section{Demographic, tumor and treatment characteristics by patient residence}

Women residing in metro counties were younger, and their counties were more likely to have low poverty and more highly educated residents than nonmetro and rural counties (Table 1). There were no clinically significant differences in stage at presentation, receptor subtype, or use of neoadjuvant chemotherapy by patient residence (results not shown).

Table 1. Patient, tumor, and treatment characteristics by patient's residence at time of diagnosis, lowa, 2007-2017 Distance traveled by patient residence and type of surgery

Rural women traveled farther than metro and nonmetro women for surgery, regardless of the type of operation (median travel distance (MTD): 6 miles metro, 25 miles nonmetro, 36 miles rural) (Fig. 3). However, the impact of type of surgery and receipt of reconstruction on travel distance differed by patient rurality. Metro women had little variation in MTD regardless of the type of operation, whereas rural and non-metro women who had reconstruction traveled the farthest distance (nonmetro MTD: 36 miles UM and CPM; rural: 49 miles UM, 51 miles CPM). Women who received lumpectomy or UM without reconstruction traveled about the same distance. Even with no reconstruction, rural and nonmetro women traveled about 7 miles farther for CPM than UM (nonmetro: 29 vs 22 miles; rural: 37 vs 30 miles).

Figure 3. Distance traveled for surgery (with/without reconstruction) for stage 0-III breast cancer by patient residence

\section{Treatment patterns of rural women by location of surgical care}

Almost one-third (29\%) of rural women underwent breast cancer surgery at a rural hospital from 2007-2017, with the proportion decreasing over the study period (Table 2). In contrast, nearly all metro (99\%) women had surgery at metro hospitals, whereas $40 \%$ of nonmetro women had surgery at nonmetro hospitals. 
Table 2

Rural patients' demographic, tumor, and treatment characteristics, stratified by surgery hospital location, lowa, 2007-2017

\begin{tabular}{|c|c|c|c|c|c|}
\hline \multirow[b]{2}{*}{ Characteristic } & \multirow[b]{2}{*}{ Surgery Hospital Location } & \multicolumn{3}{|c|}{ Rural Patient } & \multirow[t]{2}{*}{ 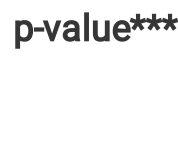 } \\
\hline & & Metro & Nonmetro & Rural & \\
\hline & TOTALS & 1842 & 781 & 1064 & \\
\hline median travel distance (miles) & & 56.3 & 24.5 & 4.5 & $<.0001$ \\
\hline \multirow[t]{4}{*}{ Age of diagnosis } & $0-39$ years & $4 \%$ & $3 \%$ & $2 \%$ & $<.0001$ \\
\hline & 40-49 year & $15 \%$ & $15 \%$ & $8 \%$ & \\
\hline & 50-59 year & $25 \%$ & $22 \%$ & $19 \%$ & \\
\hline & $60+$ years & $56 \%$ & $60 \%$ & $72 \%$ & \\
\hline \multirow[t]{2}{*}{ Race } & White & $99 \%$ & $99 \%$ & $99 \%$ & 0.63 \\
\hline & Other/Unknown & $1 \%$ & $1 \%$ & $1 \%$ & \\
\hline \multirow[t]{2}{*}{ Marital status } & Married & $72 \%$ & $65 \%$ & $58 \%$ & $<.0001$ \\
\hline & Unmarried/Unknown & $28 \%$ & $35 \%$ & $42 \%$ & \\
\hline \multirow[t]{4}{*}{ Insurance } & Private & $69 \%$ & $73 \%$ & $63 \%$ & $<.0001$ \\
\hline & Medicaid & $5 \%$ & $7 \%$ & $7 \%$ & \\
\hline & Medicare & $21 \%$ & $19 \%$ & $26 \%$ & \\
\hline & No insurance/Unknown & $5 \%$ & $1 \%$ & $5 \%$ & \\
\hline \multirow[t]{3}{*}{ Year of cancer diagnosis } & $2007-2010$ & $32 \%$ & $39 \%$ & $46 \%$ & $<.0001$ \\
\hline & $2011-2014$ & $37 \%$ & $35 \%$ & $36 \%$ & \\
\hline & $2015-2017$ & $31 \%$ & $25 \%$ & $18 \%$ & \\
\hline County poverty level & $<10 \%$ & $45 \%$ & $42 \%$ & $42 \%$ & 0.0024 \\
\hline \multirow[t]{2}{*}{ (missing = 15) } & $10-<20 \%$ & $48 \%$ & $55 \%$ & $49 \%$ & \\
\hline & $\geq 20 \%$ & $6 \%$ & $3 \%$ & $6 \%$ & \\
\hline County level bachelor & $10-19 \%$ & $71 \%$ & $95 \%$ & $64 \%$ & $<.0001$ \\
\hline \multirow[t]{2}{*}{ or higher degree } & $20-29 \%$ & $25 \%$ & $3 \%$ & $34 \%$ & \\
\hline & $30 \%+$ & $4 \%$ & $1 \%$ & $2 \%$ & \\
\hline \multirow[t]{3}{*}{ Stage } & Stage 0 & $17 \%$ & $20 \%$ & $16 \%$ & 0.047 \\
\hline & Stage I & $44 \%$ & $46 \%$ & $44 \%$ & \\
\hline & Stage II & $30 \%$ & $25 \%$ & $31 \%$ & \\
\hline \multicolumn{6}{|c|}{ *N = 3,687; 271 rural patients could not be matched to a surgery hospital and are excluded from the table. } \\
\hline \multicolumn{6}{|c|}{ **Unknown surgery category was excluded from table due to small numbers } \\
\hline$\star \star \star p$ values $<0.05$ are in bold ty & & & & & \\
\hline
\end{tabular}




\begin{tabular}{|c|c|c|c|c|c|}
\hline \multirow[b]{2}{*}{ Characteristic } & \multirow[b]{2}{*}{ Surgery Hospital Location } & \multicolumn{3}{|c|}{ Rural Patient } & \multirow[t]{3}{*}{ p-value*** } \\
\hline & & Metro & Nonmetro & Rural & \\
\hline & TOTALS & 1842 & 781 & 1064 & \\
\hline & Stage III & $9 \%$ & $8 \%$ & $10 \%$ & \\
\hline \multirow[t]{4}{*}{ Histology } & Ductal carcinoma in situ & $16 \%$ & $19 \%$ & $15 \%$ & 0.0011 \\
\hline & Invasive ductal carcinoma & $67 \%$ & $64 \%$ & $71 \%$ & \\
\hline & Invasive lobular carcinoma & $11 \%$ & $13 \%$ & $8 \%$ & \\
\hline & Other & $6 \%$ & $4 \%$ & $6 \%$ & \\
\hline \multicolumn{6}{|l|}{ Surgery Categorizations } \\
\hline \multirow[t]{3}{*}{ Surgery type } & CPM (for unilateral cancer) & $17 \%$ & $14 \%$ & $8 \%$ & $<.0001$ \\
\hline & Unilateral mastectomy & $26 \%$ & $24 \%$ & $33 \%$ & \\
\hline & Lumpectomy & $57 \%$ & $63 \%$ & $59 \%$ & \\
\hline \multirow[t]{5}{*}{ Reconstruction by surgery type } & $\mathrm{CPM}+$ reconstruction & $9 \%$ & $6 \%$ & $1 \%$ & $<.0001$ \\
\hline & CPM only & $8 \%$ & $7 \%$ & $7 \%$ & \\
\hline & UM + reconstruction & $4 \%$ & $2 \%$ & $1 \%$ & \\
\hline & UM only & $22 \%$ & $22 \%$ & $32 \%$ & \\
\hline & Lumpectomy & $57 \%$ & $63 \%$ & $59 \%$ & \\
\hline Reconstruction after any & Yes & $13 \%$ & $8 \%$ & $1 \%$ & $<.0001$ \\
\hline mastectomy & No & $87 \%$ & $92 \%$ & $99 \%$ & \\
\hline \multirow[t]{2}{*}{ CoC Accreditation } & Yes & $84 \%$ & $78 \%$ & $0 \%$ & $<.0001$ \\
\hline & No & $16 \%$ & $22 \%$ & $100 \%$ & \\
\hline \multicolumn{2}{|l|}{ Average Hospital Bed Size } & 521 & 190 & 66 & \\
\hline \multicolumn{6}{|c|}{ *N=3,687; 271 rural patients could not be matched to a surgery hospital and are excluded from the table. } \\
\hline \multicolumn{6}{|c|}{ **Unknown surgery category was excluded from table due to small numbers } \\
\hline \multicolumn{6}{|l|}{$* * * p$ values $<0.05$ are in bold type } \\
\hline
\end{tabular}

Rural women who had surgery at rural hospitals were older, more likely to be unmarried and have Medicare as primary insurance compared to other rural women. They were also much more likely to have UM without reconstruction and traveled the shortest distance for surgery (Table 2).

Overall, $50 \%$ of rural women were treated at metro hospitals. Their stage distribution was similar to those treated at rural hospitals. In contrast, rural women treated at nonmetro hospitals $(21 \%)$ tended to be diagnosed at a lower stage than those treated at other hospitals and had the highest rate of lumpectomy and the lowest rate of UM.

The rate of CPM in rural women varied widely by hospital rurality (17\% metro, $14 \%$ nonmetro, $8 \%$ rural) as did the rate of reconstruction post mastectomy (31\% metro, $23 \%$ nonmetro, $3 \%$ rural). The highest rate of reconstruction in rural women 
(57\%) was seen in those undergoing CPM at metro hospitals.

Table 2. Rural patients' demographic, tumor, and treatment characteristics, stratified by surgery hospital location, lowa, 2007-2017

\section{Interaction between hospital rurality and patient residence}

To evaluate whether differences in surgical treatment for rural patients were related solely to the hospital where they had surgery, patients treated in the same hospital location were stratified by area of residence (Table 3). Compared with nonrural women treated at metro or nonmetro hospitals, rural women were more likely to be married, have private insurance, and less likely to come from a county with high poverty. They were also more likely to have surgery at a larger, CoCaccredited or $\mathrm{NCl}$-designated institution that offered reconstruction. 
Table 3

Demographic, tumor, treatment, and surgery facility characteristics stratified by patient residence and facility location

\begin{tabular}{|c|c|c|c|c|c|c|c|c|}
\hline \multirow[t]{3}{*}{ Characteristic } & \multirow{2}{*}{$\begin{array}{l}\text { Surgery Hospital } \\
\text { Location }\end{array}$} & \multicolumn{3}{|l|}{ Metro } & \multirow[t]{3}{*}{ p-value } & \multicolumn{2}{|l|}{ Nonmetro } & \multirow{3}{*}{$\begin{array}{l}\mathrm{p}- \\
\text { value***}\end{array}$} \\
\hline & & Metro & Nonmetro & Rural & & Nonmetro & Rural & \\
\hline & TOTALS & 11579 & 3080 & 1842 & & 2073 & 781 & \\
\hline \multirow{4}{*}{$\begin{array}{l}\text { Age of } \\
\text { diagnosis }\end{array}$} & $0-39$ years & $5 \%$ & $4 \%$ & $4 \%$ & 0.006 & $2 \%$ & $3 \%$ & 0.0001 \\
\hline & 40-49 year & $17 \%$ & $16 \%$ & $15 \%$ & & $9 \%$ & $15 \%$ & \\
\hline & 50-59 year & $25 \%$ & $26 \%$ & $25 \%$ & & $20 \%$ & $22 \%$ & \\
\hline & $60+$ years & $52 \%$ & $53 \%$ & $56 \%$ & & $68 \%$ & $60 \%$ & \\
\hline \multirow[t]{2}{*}{ Race } & White & $96 \%$ & $99 \%$ & $99 \%$ & $<.0001$ & $98 \%$ & $99 \%$ & 0.15 \\
\hline & Other/Unknown & $4 \%$ & $1 \%$ & $1 \%$ & & $2 \%$ & $1 \%$ & \\
\hline \multirow[t]{2}{*}{ Marital status } & Married & $64 \%$ & $69 \%$ & $72 \%$ & $<.0001$ & $57 \%$ & $65 \%$ & 0.0002 \\
\hline & Unmarried/Unknown & $36 \%$ & $31 \%$ & $28 \%$ & & $43 \%$ & $35 \%$ & \\
\hline \multirow[t]{4}{*}{ Insurance } & Private & $67 \%$ & $65 \%$ & $69 \%$ & $<.0001$ & $51 \%$ & $73 \%$ & $<.0001$ \\
\hline & Medicaid & $6 \%$ & $6 \%$ & $5 \%$ & & $9 \%$ & $7 \%$ & \\
\hline & Medicare & $23 \%$ & $26 \%$ & $21 \%$ & & $37 \%$ & $19 \%$ & \\
\hline & $\begin{array}{l}\text { No } \\
\text { insurance/Unknown }\end{array}$ & $4 \%$ & $3 \%$ & $5 \%$ & & $3 \%$ & $1 \%$ & \\
\hline \multirow{3}{*}{$\begin{array}{l}\text { Year of cancer } \\
\text { diagnosis }\end{array}$} & $2007-2010$ & $35 \%$ & $34 \%$ & $32 \%$ & 0.05 & $41 \%$ & $39 \%$ & 0.56 \\
\hline & $2011-2014$ & $35 \%$ & $36 \%$ & $37 \%$ & & $34 \%$ & $35 \%$ & \\
\hline & $2015-2017$ & $30 \%$ & $30 \%$ & $31 \%$ & & $26 \%$ & $25 \%$ & \\
\hline $\begin{array}{l}\text { County level } \\
\text { poverty }\end{array}$ & $<10 \%$ & $62 \%$ & $53 \%$ & $45 \%$ & $<.0001$ & $39 \%$ & $42 \%$ & $<.0001$ \\
\hline \multirow[t]{2}{*}{$($ missing $=15)$} & $10-<20 \%$ & $27 \%$ & $40 \%$ & $48 \%$ & & $44 \%$ & $55 \%$ & \\
\hline & $\geq 20 \%$ & $11 \%$ & $8 \%$ & $6 \%$ & & $17 \%$ & $3 \%$ & \\
\hline $\begin{array}{l}\text { County level } \\
\text { bachelor }\end{array}$ & $10-19 \%$ & $10 \%$ & $86 \%$ & $71 \%$ & $<.0001$ & $77 \%$ & $95 \%$ & $<.0001$ \\
\hline \multirow{2}{*}{$\begin{array}{l}\text { or higher } \\
\text { degree }\end{array}$} & $20-29 \%$ & $28 \%$ & $14 \%$ & $25 \%$ & & $23 \%$ & $3 \%$ & \\
\hline & $30 \%+$ & $62 \%$ & $0 \%$ & $4 \%$ & & $0 \%$ & $1 \%$ & \\
\hline Stage & Stage 0 & $19 \%$ & $17 \%$ & $17 \%$ & 0.003 & $17 \%$ & $20 \%$ & 0.59 \\
\hline
\end{tabular}

*Due to small cell sizes metro patient numbers for nonmetro hospital were suppressed and missing data were excluded from table

**Unknown surgery category was excluded from table due to small numbers

$\star \star \star p$ value $<0.05$ are in bold type 


\begin{tabular}{|c|c|c|c|c|c|c|c|c|}
\hline \multirow[t]{6}{*}{ Characteristic } & \multirow{2}{*}{$\begin{array}{l}\text { Surgery Hospital } \\
\text { Location }\end{array}$} & \multicolumn{3}{|l|}{ Metro } & \multirow[t]{3}{*}{ p-value } & \multicolumn{2}{|l|}{ Nonmetro } & \multirow[t]{3}{*}{$\begin{array}{l}\text { p- } \\
\text { value*** }\end{array}$} \\
\hline & & Metro & Nonmetro & Rural & & Nonmetro & Rural & \\
\hline & TOTALS & 11579 & 3080 & 1842 & & 2073 & 781 & \\
\hline & Stage I & $46 \%$ & $44 \%$ & $44 \%$ & & $47 \%$ & $46 \%$ & \\
\hline & Stage II & $27 \%$ & $30 \%$ & $30 \%$ & & $27 \%$ & $25 \%$ & \\
\hline & Stage III & $8 \%$ & $8 \%$ & $9 \%$ & & $8 \%$ & $8 \%$ & \\
\hline \multirow[t]{4}{*}{ Histology } & $\begin{array}{l}\text { Ductal carcinoma in } \\
\text { situ }\end{array}$ & $18 \%$ & $16 \%$ & $16 \%$ & 0.22 & $17 \%$ & $19 \%$ & 0.22 \\
\hline & $\begin{array}{l}\text { Invasive ductal } \\
\text { carcinoma }\end{array}$ & $66 \%$ & $66 \%$ & $67 \%$ & & $64 \%$ & $64 \%$ & \\
\hline & $\begin{array}{l}\text { Invasive lobular } \\
\text { carcinoma }\end{array}$ & $11 \%$ & $12 \%$ & $11 \%$ & & $13 \%$ & $13 \%$ & \\
\hline & Other & $6 \%$ & $6 \%$ & $6 \%$ & & $6 \%$ & $4 \%$ & \\
\hline \multicolumn{9}{|l|}{$\begin{array}{l}\text { Surgery } \\
\text { Categorizations }\end{array}$} \\
\hline \multirow[t]{3}{*}{ Surgery type } & CPM & $14 \%$ & $14 \%$ & $17 \%$ & 0.007 & $7 \%$ & $14 \%$ & $<.0001$ \\
\hline & UM & $25 \%$ & $26 \%$ & $26 \%$ & & $31 \%$ & $24 \%$ & \\
\hline & Lumpectomy & $61 \%$ & $60 \%$ & $57 \%$ & & $63 \%$ & $63 \%$ & \\
\hline $\begin{array}{l}\text { Reconstruction } \\
\text { by }\end{array}$ & $\begin{array}{l}\mathrm{CPM}+ \\
\text { reconstruction }\end{array}$ & $8 \%$ & $7 \%$ & $9 \%$ & 0.010 & $2 \%$ & $6 \%$ & $<.0001$ \\
\hline \multirow[t]{4}{*}{ surgery type } & CPM only & $7 \%$ & $7 \%$ & $8 \%$ & & $5 \%$ & $7 \%$ & \\
\hline & UM + reconstruction & $4 \%$ & $4 \%$ & $4 \%$ & & $1 \%$ & $2 \%$ & \\
\hline & UM only & $21 \%$ & $22 \%$ & $22 \%$ & & $30 \%$ & $22 \%$ & \\
\hline & Lumpectomy & $61 \%$ & $60 \%$ & $57 \%$ & & $63 \%$ & $63 \%$ & \\
\hline $\begin{array}{l}\text { Reconstruction } \\
\text { after }\end{array}$ & Yes & $11 \%$ & $11 \%$ & $13 \%$ & 0.018 & $3 \%$ & $8 \%$ & $<.0001$ \\
\hline $\begin{array}{l}\text { any } \\
\text { mastectomy }\end{array}$ & No & $89 \%$ & $89 \%$ & $87 \%$ & & $97 \%$ & $92 \%$ & \\
\hline Hospital had & Yes & $99 \%$ & $99 \%$ & $99 \%$ & 0.0014 & $60 \%$ & $86 \%$ & $<.0001$ \\
\hline $\begin{array}{l}\text { reconstruction } \\
\text { services? }\end{array}$ & No & $1 \%$ & $1 \%$ & $1 \%$ & & $40 \%$ & $14 \%$ & \\
\hline \multirow{2}{*}{$\begin{array}{l}\text { CoC } \\
\text { Accreditation }\end{array}$} & Yes & $77 \%$ & $83 \%$ & $84 \%$ & $<.0001$ & $30 \%$ & $78 \%$ & $<.0001$ \\
\hline & No/Unknown & $23 \%$ & $17 \%$ & $16 \%$ & & $70 \%$ & $22 \%$ & \\
\hline \multicolumn{9}{|c|}{$\begin{array}{l}\text { *Due to small cell sizes metro patient numbers for nonmetro hospital were suppressed and missing data were } \\
\text { excluded from table }\end{array}$} \\
\hline \multicolumn{9}{|c|}{$\star \star$ Unknown surgery category was excluded from table due to small numbers } \\
\hline$\star \star *$ p value $<0.05$ & in bold type & & & & & & & \\
\hline
\end{tabular}




\begin{tabular}{|c|c|c|c|c|c|c|c|c|}
\hline \multirow[t]{3}{*}{ Characteristic } & \multirow{2}{*}{$\begin{array}{l}\text { Surgery Hospital } \\
\text { Location } \\
\text { Patient Residence }\end{array}$} & \multicolumn{3}{|l|}{ Metro } & \multirow[t]{3}{*}{ p-value } & \multicolumn{2}{|l|}{ Nonmetro } & \multirow{3}{*}{$\begin{array}{l}\text { p- } \\
\text { value }\end{array}$} \\
\hline & & Metro & Nonmetro & Rural & & Nonmetro & Rural & \\
\hline & TOTALS & 11579 & 3080 & 1842 & & 2073 & 781 & \\
\hline \multirow{2}{*}{$\begin{array}{l}\mathrm{NCl} \\
\text { Designation }\end{array}$} & Yes & $9 \%$ & $19 \%$ & $24 \%$ & $<.0001$ & $0 \%$ & $0 \%$ & - \\
\hline & No & $91 \%$ & $81 \%$ & $76 \%$ & & $100 \%$ & $100 \%$ & \\
\hline $\begin{array}{l}\text { Average } \\
\text { Hospital }\end{array}$ & & 377 & 420 & 521 & & 158 & 190 & \\
\hline \multicolumn{9}{|l|}{ Bed Size } \\
\hline \multicolumn{9}{|c|}{$\begin{array}{l}\text { *Due to small cell sizes metro patient numbers for nonmetro hospital were suppressed and missing data were } \\
\text { excluded from table }\end{array}$} \\
\hline \multicolumn{9}{|c|}{ **Unknown surgery category was excluded from table due to small numbers } \\
\hline \multicolumn{9}{|c|}{$\star \star \star p$ value $<0.05$ are in bold type } \\
\hline
\end{tabular}

The highest rate of CPM in both metro and nonmetro hospitals was seen in their rural patients (Table 3) Rural women also had the highest rate of reconstruction for both UM and CPM at these hospitals.

Table 3. Demographic, tumor, treatment, and surgery facility characteristics stratified by patient residence and facility location

\section{Multivariate analysis of factors affecting CPM}

A multivariate analysis was performed to evaluate the likelihood of CPM based on patient residence, hospital location, and travel distance (Table 4). We found that travel distance $\geq 50$ miles predicted CPM regardless of rurality. The strongest association was seen in metro patients, who were 2.3 times more likely to have CPM if traveling farther (vs. metro women who traveled $<50$ miles). Rural patients who traveled $<50$ miles were also significantly more likely to have CPM than metro women traveling $<50$ miles. The largest risk factor for CPM was younger age at diagnosis, especially for those less than age 40 vs. $60+$. CPM was also more likely for patients with stage 3 disease and invasive lobular cancer. Surgery hospital characteristics associated with CPM included metro location, reconstruction capability, and absence of $\mathrm{NCl}$ designation. $\mathrm{CoC}$ accreditation was associated with a lower likelihood of $\mathrm{CPM}(\mathrm{OR}=0.91)$ in univariate analysis, but this was not significant in the multivariate model. 
Multivariable analysis of odds of receiving a CPM (vs another surgery)

\begin{tabular}{|c|c|c|c|c|}
\hline Characteristic & & $\%$ & OR** & $\mathrm{Cl}$ \\
\hline \multirow[t]{4}{*}{ Age of diagnosis } & $0-39$ years & $4 \%$ & 7.28 & $(5.97,8.88)$ \\
\hline & 40-49 year & $15 \%$ & 3.80 & $(3.29,4.40)$ \\
\hline & 50-59 year & $24 \%$ & 2.11 & $(1.84,2.43)$ \\
\hline & $60+$ years & $56 \%$ & 1.00 & REF \\
\hline \multirow[t]{2}{*}{ Marital status } & Married & $65 \%$ & 1.00 & REF \\
\hline & Unmarried/Unknown & $35 \%$ & 0.92 & $(0.82,1.03)$ \\
\hline \multirow[t]{4}{*}{ Insurance } & Private & $65 \%$ & 1.00 & REF \\
\hline & Medicaid & $7 \%$ & 1.03 & $(0.85,1.26)$ \\
\hline & Medicare & $26 \%$ & 0.73 & $(0.62,0.87)$ \\
\hline & No insurance/Unknown & $3 \%$ & 0.97 & $(0.73,1.31)$ \\
\hline \multirow[t]{3}{*}{ Year of cancer diagnosis } & 2007-2010 & $15 \%$ & 1.00 & REF \\
\hline & $2011-2014$ & $47 \%$ & 1.74 & $(1.48,2.05)$ \\
\hline & $2015-2017$ & $38 \%$ & 1.19 & $(1.00,1.40)$ \\
\hline \multirow[t]{3}{*}{ County level poverty } & $<10 \%$ & $55 \%$ & 1.00 & REF \\
\hline & $10-<20 \%$ & $35 \%$ & 0.79 & $(0.70,0.89)$ \\
\hline & $\geq 20 \%$ & $10 \%$ & 0.83 & $(0.70,1.00)$ \\
\hline \multirow[t]{4}{*}{ Stage } & Stage 0 & $18 \%$ & 0.77 & $(0.41,1.42)$ \\
\hline & Stage I & $46 \%$ & 0.57 & $(0.51,0.65)$ \\
\hline & Stage II & $28 \%$ & 1.00 & REF \\
\hline & Stage III & $8 \%$ & 1.64 & $(1.39,1.93)$ \\
\hline \multirow[t]{4}{*}{ Histology } & Ductal carcinoma in situ & $17 \%$ & 0.73 & $(0.39,1.38)$ \\
\hline & Invasive ductal carcinoma & $66 \%$ & 1.00 & REF \\
\hline & Invasive lobular carcinoma & $11 \%$ & 1.50 & $(1.29,1.75)$ \\
\hline & Other & $5 \%$ & 0.90 & $(0.69,1.17)$ \\
\hline \multirow[t]{3}{*}{ Hospital location } & Metro & $82 \%$ & 1.00 & REF \\
\hline & Nonmetro & $14 \%$ & 0.73 & $(0.59,0.90)$ \\
\hline & Rural & $4 \%$ & 0.62 & $(0.43,0.91)$ \\
\hline Hospital had reconstruction services? & Yes & $93 \%$ & 2.30 & $(1.65,3.21)$ \\
\hline
\end{tabular}

${ }^{*} N=15,299 ; 6859$ cases who did not have hospital surgery, geocode, or poverty data were excluded from model

** Adjusted for all other variables in the table; $p$ value $<0.05$ are in bold type 


\begin{tabular}{|c|c|c|c|c|}
\hline \multicolumn{2}{|l|}{ Characteristic } & \multirow{2}{*}{$\begin{array}{l}\% \\
7 \%\end{array}$} & \multirow{2}{*}{$\begin{array}{l}\text { OR** } \\
1.00\end{array}$} & \multirow{2}{*}{$\begin{array}{l}\mathrm{Cl} \\
\mathrm{REF}\end{array}$} \\
\hline & No & & & \\
\hline \multirow[t]{2}{*}{$\mathrm{NCl}$ Designation } & Yes & $11 \%$ & 1.00 & REF \\
\hline & No & $89 \%$ & 2.34 & $(1.92,2.86)$ \\
\hline Interaction term: Patient residence $\mathrm{x}$ & Metro patient who traveled $<50$ miles & $56 \%$ & 1.00 & REF \\
\hline \multirow[t]{5}{*}{ travel distance to surgery facility } & Nonmetro patient who traveled $<50$ miles & $22 \%$ & 1.06 & $(0.91,1.23)$ \\
\hline & Rural patient who traveled $<50$ miles & $10 \%$ & 1.29 & $(1.03,1.61)$ \\
\hline & Metro patient who traveled $>=50$ miles & $2 \%$ & 2.34 & $(1.69,3.23)$ \\
\hline & Nonmetro patient who traveled $>=50$ miles & $4 \%$ & 1.49 & $(1.14,1.93)$ \\
\hline & Rural patient who traveled $>=50$ miles & $6 \%$ & 1.43 & $(1.15,1.77)$ \\
\hline \multicolumn{5}{|c|}{${ }^{\star} N=15,299 ; 6859$ cases who did not have hospital surgery, geocode, or poverty data were excluded } \\
\hline \multicolumn{5}{|l|}{ from model } \\
\hline ** Adjusted for all other variables in & able; $p$ value $<0.05$ are in bold type & & & \\
\hline
\end{tabular}

Table 4. Multivariable analysis of odds of receiving a CPM (vs another surgery)

\section{Discussion:}

lowa was reported to have the second highest proportion of CPM among women under 45 years of age between 20042012 [8]. In the same study, three adjoining upper Midwestern states (South Dakota, Missouri, Nebraska) as well as 4 other states (Colorado, Tennessee, Maine, Montana) had proportions of CPM exceeding $40 \%$ in this age group. We postulated that the treatment patterns identified in lowa may also be present in other states and could be useful in understanding this geographical variation in CPM use. It is well-established that CPM is more common in affluent, educated women who more typically reside in metro areas [25], but the contribution of rurality to CPM has not been previously studied. The percent population living in rural areas in the above 8 states ranged from 14-61\%, with a median of $35 \%$ [22]. In this study, we found that the high proportion of CPM in lowa was partially the result of young rural women undergoing this procedure at a higher rate than the national levels in SEER, indeed at proportions higher than that of metro lowa women. This higher proportion of CPM in rural lowa women is unexpected and may be the result of rural health care disparities.

About one-fifth of the population of lowa lives in areas with a population density of fewer than 20,000 people [20, 22]. In rural counties, there were no $\mathrm{CoC}$-accredited hospitals, and breast reconstruction was rarely performed. Many lowa rural counties are adjacent to more populous nonmetro areas (Fig. 1), however most nonmetro hospitals also did not perform breast reconstruction or have $\mathrm{CoC}$ accreditation. Although multidisciplinary cancer care is not exclusive to CoCaccredited facilities, in lowa it has been shown to correlate with more comprehensive services [26]. It is therefore not surprising that only $20 \%$ of rural women with breast cancer were treated at rural hospitals from 2015-2017, and that half of nonmetro women had surgery at metro hospitals instead.

Rural patients traveled the farthest for their surgical care, regardless of the type of surgery they had. Over one-third traveled more than 50 miles for surgery, and despite making up only $18 \%$ of our cohort, rural patients account for about half of all the patients that traveled > 50 miles. Travel distance was an independent predictor of CPM, although the effect size was determined by where a woman lived. Both metro and nonmetro women who traveled $>50$ miles for surgery 
were more likely to have CPM than those who did not. In contrast, compared to the $96 \%$ of metro patients who traveled < 50 miles for surgery, rural patients were more likely to undergo CPM regardless of travel distance. This may be because even those rural women who traveled $<50$ miles had a longer median travel distance than metro and nonmetro patients in the $<50$ mile category, as they were often driving to hospitals in a different community (data not shown).

Travel distance for these rural patients likely does not reflect the closest institution providing surgical care for breast cancer. Rural lowans have previously demonstrated a willingness to travel farther to seek a higher level of cancer care [26]. The greatest travel distances were noted in both CPM and UM patients with reconstruction, and the desire for reconstruction may explain some of these patterns [27]. However, only half of all CPM patients ultimately received reconstruction, and the relationship between travel distance and CPM persisted even after adjusting for reconstruction, just as it did in the NCDB study [7]. CPM is associated with a more active decision-making role for surgery [28] and greater likelihood of seeking a second opinion [29], which may be reflected in a willingness to travel for care. Rural women tended to seek out larger metro hospitals and those that were CoC-accredited, or NCl-designated. They also had higher proportion of CPM use than other women of similar ages treated at those hospitals, implying an intrinsic preference for CPM in this group. Women facing a high travel burden for the level of care they desire may perceive CPM as one way to minimize future need for healthcare utilization, particularly if they are rural or of lower socioeconomic status $[16,30]$.

There were other important contributors to the high rate of CPM in lowa. For metro patients, who accounted for $61 \%$ of CPM cases, travel distance was less important, as only a small subset (4\%) of metro women traveled $>50$ miles, and in general there was little variability in travel distance by surgery type in this population (Fig. 3). The high CPM rates in this group likely relate to the more well described factors of age, race, income, education, insurance and access to reconstruction $[2,7,8,25]$. Younger age was the strongest predictor of CPM for women across lowa and has been consistently associated with CPM in the literature $[2,7,8]$, although age alone is not considered an indication for CPM [3, $5,31]$. The proportion of women under 40 years undergoing CPM for metro and nonmetro was high (40 and $39 \%)$. However, the highest proportion was seen in rural women in this age group, with over half undergoing CPM. This may reflect the additive effect of age and travel distance in this population. Additionally, we found that both lobular carcinoma and stage 3 disease were associated with CPM, a phenomenon also reported in SEER [2]. Since expert consensus is that lobular carcinoma is not an indication for CPM and prophylactic surgery would provide even less benefit than usual in higher stage disease [5], it is unclear whether these patients are being recommended CPM inappropriately or are being incompletely counseled about risks and benefits. Fortunately, the rate of CPM in lowa has been falling in all populations since 2013 and in 2015 was similar to or below national rates, which also appear to be leveling off.

Our study has several limitations. The lowa Cancer Registry does not capture everything that can impact hospital selection or surgical decision making, such as genetic mutation, family history, additional testing or number of visits [32-34]. lowa has a predominantly White population with few non-Whites residing in rural areas, so we were unable to examine the interaction of race and rurality. In addition, because our analysis was restricted to one state, it makes generalization of our findings beyond the upper Midwest difficult. However, restricting analysis to one state's cancer registry, combined with our familiarity with local communities, ensures that our definitions of rurality reflect clinically significant differences in populations and resources. This is especially relevant considering the existing variability in how rurality is defined [35]. Additionally, a strength of the lowa Cancer Registry is that it includes facilities of all types and sizes, unlike the NCDB that only includes CoC-accredited hospitals. A recent study evaluating rural urban differences in the National Cancer database failed to show much difference in the use of CPM by rurality when analysis was restricted only to $\mathrm{CoC}$-accredited hospitals. We found $\mathrm{CoC}$ accreditation was associated with a lower likelihood of CPM, although this finding did not meet statistical significance. [13]. 


\section{Conclusions}

Our findings suggest a disparity in surgical care for rural lowan women with breast cancer, for whom travel distance may be dictated not just by the distance to the nearest hospital but rather to the nearest hospital offering reconstruction and multidisciplinary care. In lowa, it appears that many rural women are willing to travel farther to access this kind of care, with the potential for significant socioeconomic burden and less than optimal care. Counseling for patients desiring CPM should take into consideration rurality, access to local healthcare, expectations regarding subsequent visits for surveillance and completion of reconstruction, and measures to make follow-up care less burdensome.

\section{List of abbreviations}

\begin{tabular}{|ll|}
\hline CPM & Contralateral prophylactic mastectomy \\
\hline UM & Unilateral mastectomy \\
\hline MTD & Median travel distance \\
\hline SEER & Surveillance, Epidemiology, and End Results \\
\hline CoC & Commission on Cancer \\
\hline NCDB & National Cancer Database \\
\hline RUCC & Rural Urban Continuum Codes \\
\hline
\end{tabular}

\section{Declarations}

\section{Declarations}

Ethics approval and consent to participate: This study was granted human subject exemption status by the University of lowa Institutional Review Board.

\section{Consent for publication: Not applicable}

Availability of data and materials: Cases were extracted from the lowa Cancer Registry Surveillance, Epidemiology, and End Results (SEER) Data Management System (SEER*DMS).). The datasets generated and/or analyzed during the current study are not publicly available because they contain protected health information of patients, as well as specific names of hospitals. A de-identified version of our analytic dataset could be available from the corresponding author upon reasonable request.

Competing interests: None of the authors have any financial or non-financial competing interests

Funding: This work was supported by the National Cancer Institute at the National Institutes of Health (grant numbers HHSN261201800012I/ HHSN26100001 to CFL, MEC, ARK; P30 CA086862 to MEC, ARK).

Author's contributions: AK performed primary data analysis and prepared the methods section, tables and figures. EJ assisted in the primary data analysis, literature review and preparation of the manuscript. MC and CL provided expertise in the datasets used, directed the statistical analysis and contributed to the writing of the manuscript. IL and SS were involved in developing study design. IL was the primary manuscript author and SS was a major contributor in writing the manuscript. All authors read, edited and approved the final manuscript. 


\section{References}

1. Fisher B, Anderson S, Bryant J, Margolese RG, Deutsch M, Fisher ER, Jeong J-H, Wolmark N. Twenty-Year Follow-up of a Randomized Trial Comparing Total Mastectomy, Lumpectomy, and Lumpectomy plus Irradiation for the Treatment of Invasive Breast Cancer. N Engl J Med. 2002;347(16):1233-41.

2. Wong SM, Freedman RA, Sagara Y, Aydogan F, Barry WT, Golshan M. Growing Use of Contralateral Prophylactic Mastectomy Despite no Improvement in Long-term Survival for Invasive Breast Cancer. Ann Surg. 2017;265(3):5819.

3. Pesce C, Liederbach E, Wang C, Lapin B, Winchester DJ, Yao K. Contralateral prophylactic mastectomy provides no survival benefit in young women with estrogen receptor-negative breast cancer. Ann Surg Oncol. 2014;21(10):32319.

4. Boughey JC, Attai DJ, Chen SL, Cody HS, Dietz JR, Feldman SM, Greenberg CC, Kass RB, Landercasper J, Lemaine V, et al. Contralateral Prophylactic Mastectomy (CPM) Consensus Statement from the American Society of Breast Surgeons: Data on CPM Outcomes and Risks. Ann Surg Oncol. 2016;23(10):3100-5.

5. Hunt KK, Euhus DM, Boughey JC. al e: Society of Surgical Oncology Breast Disease Working Group Statement on Prophylactic (Risk-Reducing) Mastectomy. Ann Surg Oncol. 2017;24(2):375-97.

6. Wright FC, Look Hong NJ, Quan ML, Beyfuss K, Temple S, Covelli A, Baxter N, Gagliardi AR. Indications for Contralateral Prophylactic Mastectomy: A Consensus Statement Using Modified Delphi Methodology. Ann Surg. 2018;267(2):271-9.

7. Ward EP, Unkart JT, Bryant A, Murphy J, Blair SL. Influence of Distance to Hospital and Insurance Status on the Rates of Contralateral Prophylactic Mastectomy, a National Cancer Data Base study. Ann Surg Oncol. 2017;24(10):3038-47.

8. Nash R, Goodman M, Lin CC, Freedman RA, Dominici LS, Ward K, Jemal A. State Variation in the Receipt of a Contralateral Prophylactic Mastectomy Among Women Who Received a Diagnosis of Invasive Unilateral EarlyStage Breast Cancer in the United States, 2004-2012. JAMA Surg. 2017;152(7):648-57.

9. Meit M, Knudson A, Gilbert T, et al. The 2014 Update of the Rural-Urban Chartbook. Bethesda, MD: Rural Health Reform Policy Research Center Web site. https://ruralhealth.und.edu/projects/health-reform-policy-researchcenter/pdf/2014-rural-urban-chartbook-update.pdf.Published 2014. Accessed June 30, 2020.

10. Weaver KE, Palmer N, Lu L, Case LD, Geiger AM. Rural-urban differences in health behaviors and implications for health status among US cancer survivors. Cancer Causes Control. 2013;24(8):1481-90.

11. Iglehart JK. The challenging quest to improve rural health care. N Engl J Med. 2018;378(5):473-9.

12. Henley SJ, Anderson RN, Thomas CC, Massetti GM, Peaker B, Richardson LC. Invasive Cancer Incidence, 20042013, and Deaths, 2006-2015, in Nonmetropolitan and Metropolitan Counties - United States. MMWR Surveill Summ. 2017;66(14):1-13.

13. Obeng-Gyasi S, Timsina L, Bhattacharyya O, Fisher CS, Haggstrom DA. Breast Cancer Presentation, Surgical Management and Mortality Across the Rural-Urban Continuum in the National Cancer Database. Ann Surg Oncol. 2020;27(6):1805-15.

14. Lin CC, Bruinooge SS, Kirkwood MK, Olsen C, Jemal A, Bajorin D, Giordano SH, Goldstein M, Guadagnolo BA, Kosty $M$, et al. Association Between Geographic Access to Cancer Care, Insurance, and Receipt of Chemotherapy: Geographic Distribution of Oncologists and Travel Distance. J Clin Oncol. 2015;33(28):3177-85. 
15. Lin Y, Wimberly MC, Da Rosa P, Hoover J, Athas WF. Geographic access to radiation therapy facilities and disparities of early-stage breast cancer treatment. Geospat Health. 2018;13(1):622.

16. Polacek GN, Ramos MC, Ferrer RL. Breast cancer disparities and decision-making among U.S. women. Patient Educ Couns. 2007;65(2):158-65.

17. Meden T, St John-Larkin C, Hermes D, Sommerschield S. Relationship between travel distance and utilization of breast cancer treatment in rural northern Michigan. JAMA. 2002;287(1):111.

18. Goyal S, Chandwani S, Haffty BG, Demissie K. Effect of travel distance and time to radiotherapy on likelihood of receiving mastectomy. Ann Surg Oncol. 2015;22(4):1095-101.

19. Freedman RA, Virgo KS, He Y, et al: The association of race/ethnicity, insurance status, and socioeconomic factors with breast cancer care. Cancer; 2011:117 (1):180-9.

20. United States Department of Agriculture Rural-Urban Continuum Codes. https://www.ers.usda.gov/dataproducts/rural-urban-continuum-codes/. Published 2016. Accessed June 30, 2020.

21. Bennett T, Skatrud JD, Guild P, Loda F, Klerman LV. Rural adolescent pregnancy: a view from the South. Fam Plann Perspect. 1997;29(6):256-60, 267.

22. Census Bureau US. 2012-2016 American Community Survey 5-Year Estimates. https://www.census.gov/programssurveys/acs/technical-documentation/table-and-geography-changes/2016/5-year.html. Accessed June 30, 2020.

23. SEER Program Coding and Staging Manual. 2016: Surgery Codes Breast.

https://seer.cancer.gov/archive/manuals/2016/AppendixC/Surgery_Codes_Breast_2016.pdf. Accessed June30, 2020.

24. Surveillance E, Results E (SEER) Program (www.seer.cancer.gov) SEER*Stat Database: Incidence - SEER Research Plus Data, 18 Registries, Nov 2019 Sub (2000-2017) - Linked To County Attributes - Total U.S., 1969-2018 Counties, National Cancer Institute, DCCPS, Surveillance Research Program, released April 2020, based on the November 2019 submission. Accessed June 30, 2020.

25. Grimmer L, Liederbach E, Velasco J, Pesce C, Wang CH, Yao K. Variation in Contralateral Prophylactic Mastectomy Rates According to Racial Groups in Young Women with Breast Cancer, 1998 to 2011: A Report from the National Cancer Data Base. J Am Coll Surg. 2015;221(1):187-96.

26. Ward MM, Ullrich F, Matthews K, Rushton G, Tracy R, Goldstein MA, Bajorin DF, Kosty MP, Bruinooge SS, Hanley A, et al. Where do patients with cancer in lowa receive radiation therapy? J Oncol Pract. 2014;10(1):20-5.

27. Albornoz CR, Cohen WA, Razdan SN, Mehrara BJ, McCarthy CM, Disa JJ, Dayan JH, Pusic AL, Cordeiro PG, Matros E. The Impact of Travel Distance on Breast Reconstruction in the United States. Plast Reconstr Surg. 2016;137(1):12-8.

28. Rosenberg SM, Sepucha K, Ruddy KJ, Tamimi RM, Gelber S, Meyer ME, Schapira L, Come SE, Borges VF, Golshan M, et al. Local Therapy Decision-Making and Contralateral Prophylactic Mastectomy in Young Women with Early-Stage Breast Cancer. Ann Surg Oncol. 2015;22(12):3809-15.

29. Bellavance E, Peppercorn J, Kronsberg S, Greenup R, Keune J, Lynch J, Collyar D, Magder L, Tilburt J, Hlubocky F, et al. Surgeons' Perspectives of Contralateral Prophylactic Mastectomy. Ann Surg Oncol. 2016;23(9):2779-87.

30. Baptiste DF, MacGeorge EL, Venetis MK, Mouton A, Friley LB, Pastor R, Hatten K, Lagoo J, Clare SE, Bowling MW. Motivations for contralateral prophylactic mastectomy as a function of socioeconomic status. BMC Womens Health. 2017;17(1):10.

31. Roberts A, Habibi M, Frick K: Cost-effectiveness of contralateral prophylactic mastectomy for prevention of contralateral breast cancer. In., vol. 21. Ann Surg Oncol; 2014: 2209-17.

32. Reiner AS, John EM, Brooks JD, Lynch CF, Bernstein L, Mellemkjær L, Malone KE, Knight JA, Capanu M, Teraoka SN, et al. Risk of asynchronous contralateral breast cancer in noncarriers of BRCA1 and BRCA2 mutations with a family 
history of breast cancer: a report from the Women's Environmental Cancer and Radiation Epidemiology Study. J Clin Oncol. 2013;31(4):433-9.

33. Reiner AS, Lynch CF, Sisti JS, John EM, Brooks JD, Bernstein L, Knight JA, Hsu L, Concannon P, Mellemkjær L, et al. Hormone receptor status of a first primary breast cancer predicts contralateral breast cancer risk in the WECARE study population. Breast Cancer Res. 2017;19(1):83.

34. Lizarraga IM, Sugg SL, Weigel RJ, Scott-Conner CE. Review of risk factors for the development of contralateral breast cancer. Am J Surg. 2013;206(5):704-8.

35. Black P. Wisconsin Divided Six Ways: A Review of Rural-Urban Classification Systems.

[http://worh.org/sites/default/files/Wisconsin\%20Divided\%20Six\%20Ways_1.pdf]Published 2016. Accessed June 30, 2020.

\section{Figures}

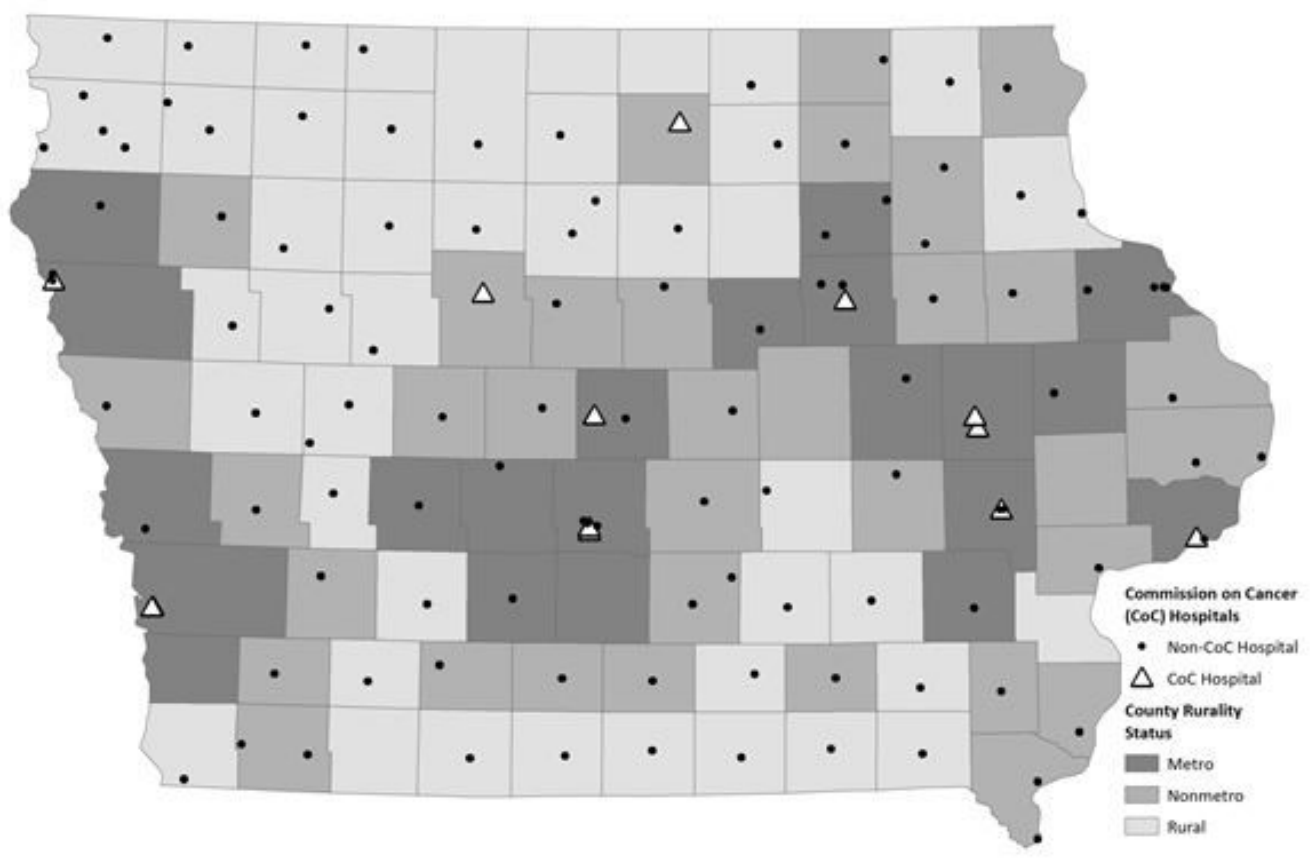

\section{Figure 1}

Map of lowa Hospitals and County Rurality 


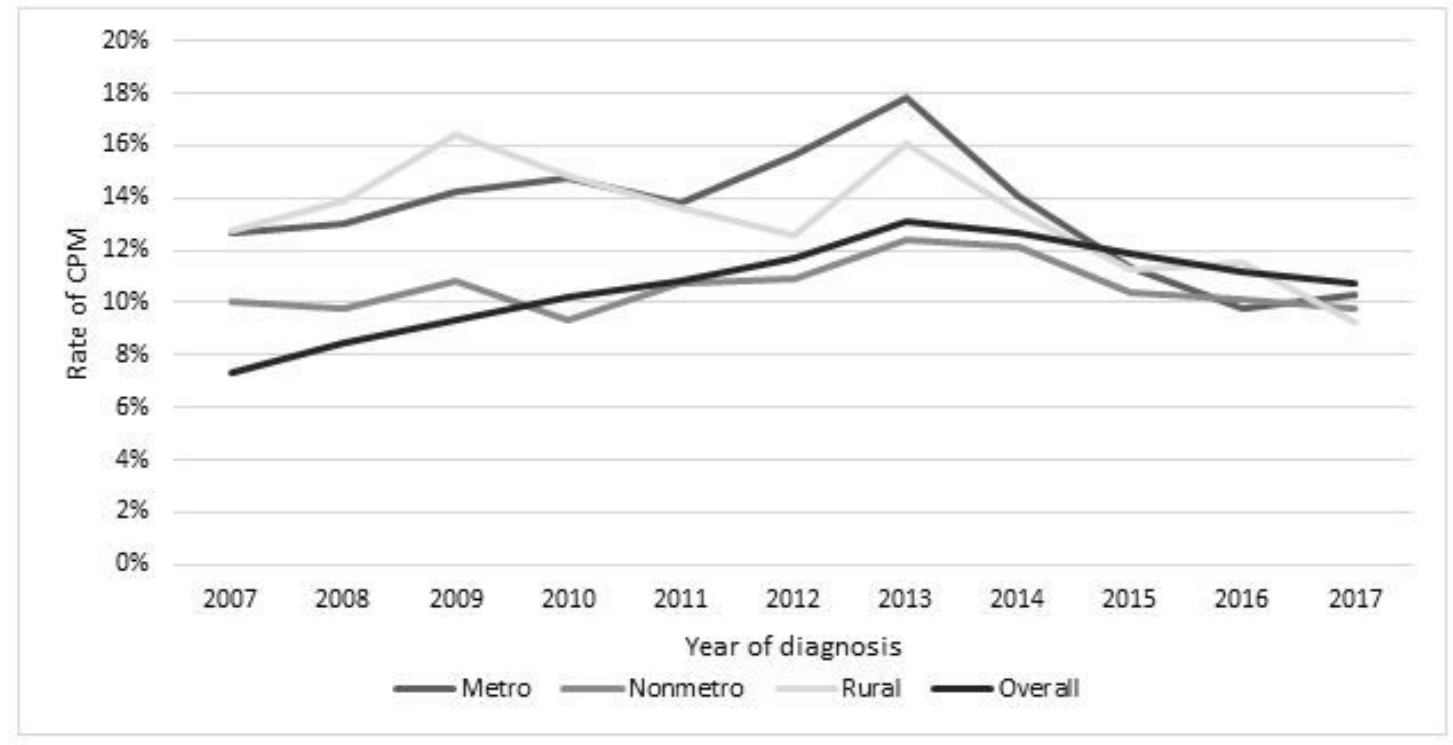

Figure 2

Proportion of CPM by patient residence for stage 0 -III breast cancer, lowa vs other SEER18

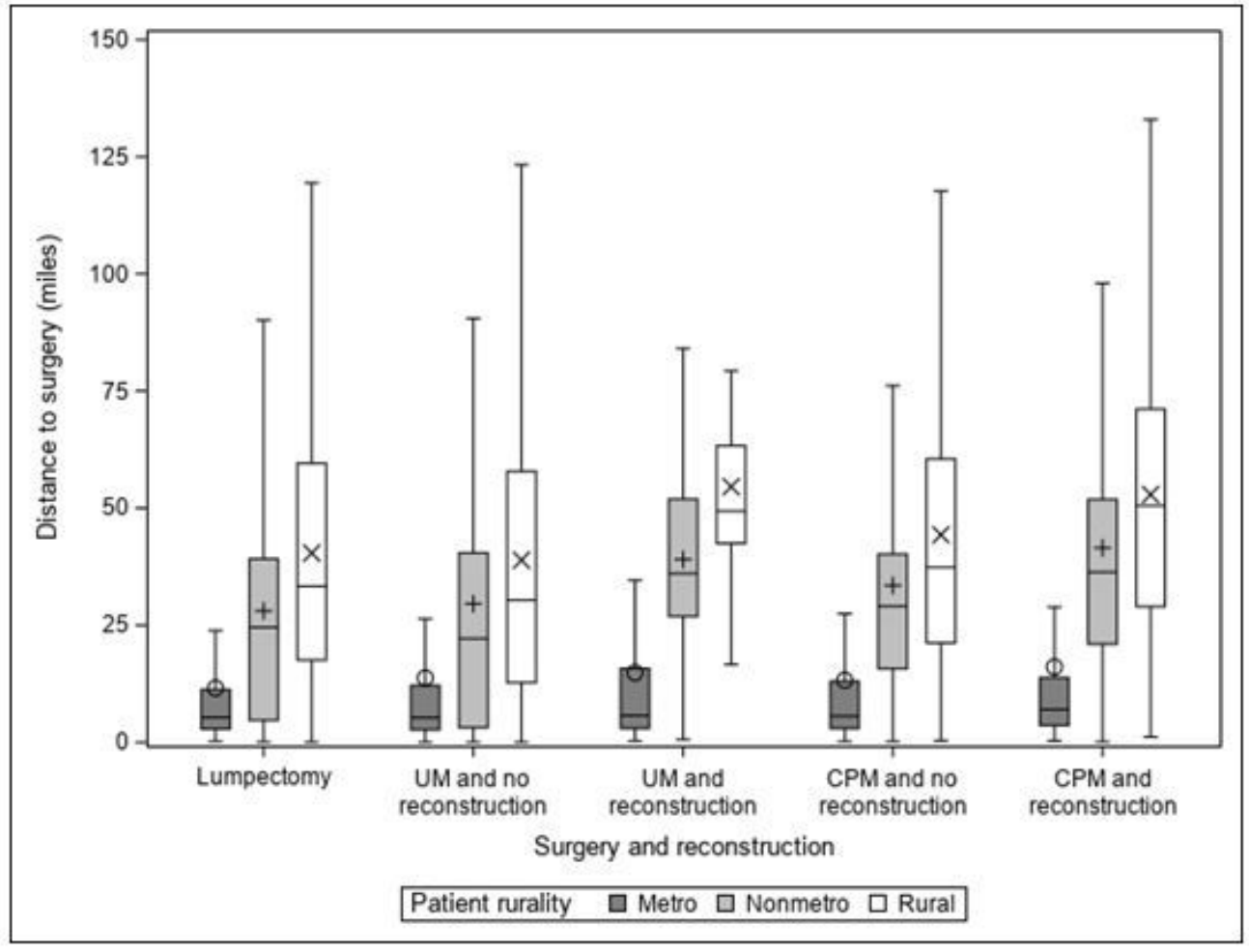

Figure 3

Distance traveled for surgery (with/without reconstruction) for stage 0 -III breast cancer by patient residence *Circle, plus, and cross represent mean distance; Whiskers extend to 1.5 (IQR) above/below Q1/Q3 